\title{
A high-resolution full-field range imaging system
}

\author{
D. A. Carnegie, ${ }^{\text {a) }}$ M. J. Cree, and A. A. Dorrington \\ Department of Physics and Electronic Engineering, University of Waikato, Hamilton, New Zealand
}

(Received 7 February 2005; accepted 30 May 2005; published online 21 July 2005)

\begin{abstract}
There exist a number of applications where the range to all objects in a field of view needs to be obtained. Specific examples include obstacle avoidance for autonomous mobile robots, process automation in assembly factories, surface profiling for shape analysis, and surveying. Ranging systems can be typically characterized as being either laser scanning systems where a laser point is sequentially scanned over a scene or a full-field acquisition where the range to every point in the image is simultaneously obtained. The former offers advantages in terms of range resolution, while the latter tend to be faster and involve no moving parts. We present a system for determining the range to any object within a camera's field of view, at the speed of a full-field system and the range resolution of some point laser scans. Initial results obtained have a centimeter range resolution for a 10 second acquisition time. Modifications to the existing system are discussed that should provide faster results with submillimeter resolution. () 2005 American Institute of Physics.
\end{abstract}

[DOI: $10.1063 / 1.1988312]$

\section{INTRODUCTION}

There are many applications where the precise range to an object is required. Surveying, autonomous mobile robotics, automated factory processes, medical imaging, shape reconstruction, vehicle crash scene investigations, and sail profile determination for racing yachts are a few of the potential uses of a distance ranging system. Each of these applications has their own requirements in terms of range resolution and acquisition time. We present in this paper a full-field distance ranging system eventually capable of submillimeter range resolution independent of the texture, color and orientation of the imaged objects.

Image ranging systems can be usefully classified by their acquisition hardware as being either laser point scanning or full-field (simultaneous) image acquisition. The high precision ranging and $x-y$ positioning of the laser scanner is obtained by physically moving a laser dot over a field of interest. The movement of the laser dot necessitates the inclusion of moving parts, and it can take a significant length of time to entirely cover the field of view while maintaining high precision. It is commonly necessary to employ software interpolation and edge detection algorithms in order to obtain a clear image of the features in the area of interest, with a corresponding further increase in range determination time and potential blurring of object resolution. Full-field techniques do not suffer the time costs of the laser point scanning and can be configured to operate in near real time. Also they do not suffer the inconvenience of requiring moving parts. However, such systems do incur a penalty in terms of exhibiting a significant loss of resolution and $x-y$ positioning compared to the laser scheme.

Image ranging systems can also be classified by the ranging method used, the most common being direct time-

${ }^{a)}$ Electronic mail: phys2236@waikato.ac.nz of-flight measurement, AM/FM modulation, structured lighting, and time of flight measurements of picosecond pulses. ${ }^{1}$ These methods are compared in Table I.

As structured lighting systems are primarily for shape reconstruction and are often limited to continuous surfaces, they are of limited usefulness for the more generic ranging applications we are designing for and we do not consider them any further. Evaluation of the flight time of the picosecond laser pulses provides a range resolution of the order of a few millimeters for ranges between a few hundred meters up to several thousand kilometers (satellite laser ranging). Such a system operates outside the range bounds considered by this paper, so is also not further considered. Table II lists some known research and commercial ranging systems and where they fit in with the classifications introduced above. This table also presents details on the total operating range, the range resolution achieved and the fundamental limitation to range resolution. Also listed is the field-of-view (FOV) $x-y$ resolution achievable within the FOV and the time to collect information over the whole FOV. There are two entries for the $Z \& F$ laser scanner as it has two range modes. The abbreviation pps refers to pixels per second and fps is frames per second.

Comparing full-field simultaneous imaging against laser scanning it is clear that full-field imaging has the greatest potential for high speed data acquisition. It is also clear that $\mathrm{AM} / \mathrm{FM}$ modulation gives the best ranging resolution, however, it should be realized that this advantage is not due to any fundamental limitation on time-of-flight but is due to limitations on the speed of operation of current electronic circuitry. This advantage with $\mathrm{AM} / \mathrm{FM}$ modulation is not so apparent with the full-field rangers because all commercial full-field rangers surveyed that use AM modulation adopt a homodyne approach to encode range into the intensity re- 
TABLE I. Ranging methods.

\begin{tabular}{ll}
\hline \hline Ranging method & \multicolumn{1}{c}{ Features } \\
\hline Time of flight & $\begin{array}{l}\text { Potentially high range resolution } \\
\text { Modulated light source usually laser diode } \\
\text { Requires very high speed and carefully } \\
\text { engineered electronics }\end{array}$ \\
& High cost \\
& Potentially very high range resolution \\
& Modulated light source usually laser diode \\
LM/FM modulation & Less strident requirements on electronics \\
& Lower cost than time of flight \\
Structured lighting & Lowest range resolution \\
& Limited range-small objects \\
& Simple hardware and lighting \\
Licosecond laser & Lowest cost \\
pulse timing & Time-of-flight measurement \\
& High range resolution \\
& Long-distance measurements \\
& Very high cost \\
\hline \hline
\end{tabular}

ceived by the camera. Digital cameras have limited dynamic resolution and this impacts severely on the determination of the intensity of the light.

It is also clear from Table II that the Leica and Z\&F rangers are superior to other commercial systems when considering the resolution of ranging and $x-y$ positioning. The complexity and bulk of a laser scanner however, translates into increased cost and is not suitable for certain applications. There is a place for full-field ranging which explains the presence of commercial full-field rangers on the market. Initial tests indicate that our system has a superior range resolution compared to the currently available full-field rangers, and it is nearing the range resolution of the $\mathrm{Z} \& \mathrm{~F}$ ranger. Currently it is not as fast as the lower range resolution fullfield imagers detailed in Table II, however, the system described in this paper can be reconfigured in software and

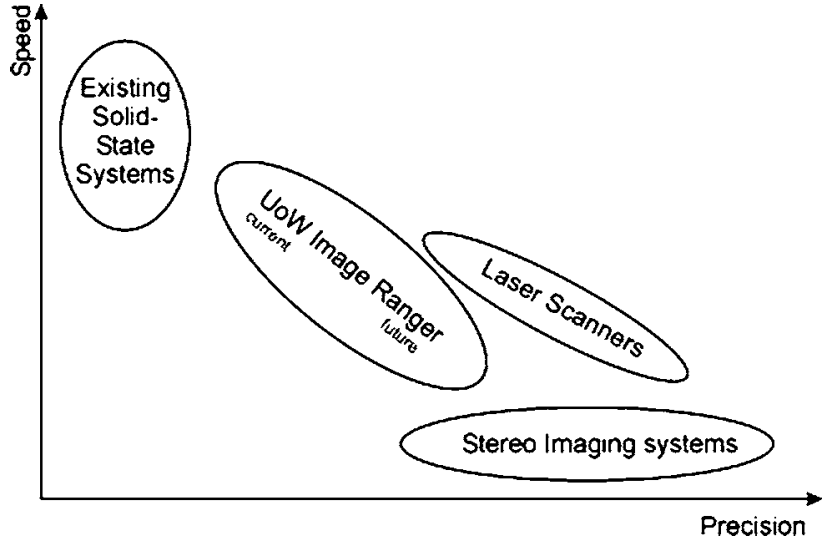

FIG. 1. Ranging system technology—-speed versus precision.

firmware to provide faster measurements at the cost of precision. Diagrammatically, the niche this ranging system fills can be seen in Fig. 1.

\section{IMAGE RANGING}

Most modern full-field rangers that we know of (for example, Refs. 7-10) use a modulated light signal which when reflected back from the scene is interrupted by a high-speed shutter before impinging on the camera (see Fig. 2). The time-of-flight $\tau$ is encoded into a phase shift $\phi$ that describes the retardation of the received light signal behind the transmitted light signal. Taking the light source as coincident with the camera then

$$
\tau=\frac{2 d}{c} \text { and } \phi=2 \pi f t=\frac{4 \pi f d}{c},
$$

where $d$ is the camera-object distance, $f$ is the frequency of the modulation of the transmitted light, and $c$ is the speed of light. The received light signal on entering the high speed shutter is mixed with the same signal as the transmitted light which has the effect of encoding the phase shift $\phi$ (and hence the range) into some other component (such as intensity) of the signal at the camera. Most importantly, the signal result-

TABLE II. Image ranger specifications.

\begin{tabular}{|c|c|c|c|c|c|c|c|c|}
\hline System & Acquisition & $\begin{array}{l}\text { Ranging } \\
\text { method }\end{array}$ & $\begin{array}{l}\text { Range } \\
(\mathrm{m})\end{array}$ & $\begin{array}{l}\text { Range } \\
\text { resolution } \\
(\mathrm{mm})\end{array}$ & $\begin{array}{l}\text { Range } \\
\text { resolution } \\
\text { limit }\end{array}$ & $\begin{array}{l}\text { FOV } \\
\text { (pixels) }\end{array}$ & $\begin{array}{c}X Y \\
\text { resolution } \\
(\mathrm{mm})\end{array}$ & $\begin{array}{l}\text { Acquisition } \\
\text { time }\end{array}$ \\
\hline Cyrax & Laser & Time of flight & $1.5-50$ & 4 & Electronic speed & Scanner & 6 & $1 \mathrm{kpps}$ \\
\hline Leica LR200 & & & $0-35$ & 0.05 & Electronic speed & Scanner & 0.3 & $1 \mathrm{kpps}$ \\
\hline $\mathrm{Z} \& \mathrm{~F}$ & Laser & $\mathrm{AM} / \mathrm{FM}$ & $0.4-25.2$ & 0.38 & Dynamic range (16-bit) & Scanner & 0.8 & $125 \mathrm{kpps}$ \\
\hline $\mathrm{Z} \& \mathrm{~F}$ & Laser & $\mathrm{AM} / \mathrm{FM}$ & $0.4-53.5$ & 0.82 & & & 1.7 & \\
\hline Z-Cam (Ref. 2) & Full field & $\begin{array}{l}\text { Gated time } \\
\text { of flight }\end{array}$ & $0.5-7$ & 10 & Dynamic range & $720 \times 582$ & $6.94 \mathrm{~mm}^{\mathrm{a}}$ & $60 \mathrm{fps}$ \\
\hline Zmini (Ref. 3) & Full field & $\begin{array}{l}\text { Gated time } \\
\text { of flight }\end{array}$ & $0.5-3.5$ & $\begin{array}{l}15 \text { full range, } \\
5 \text { over } 0.5 \mathrm{~m}\end{array}$ & Dynamic range (8-bit) & $768 \times 494$ & $6.51 \mathrm{~mm}^{\mathrm{a}}$ & $30 \mathrm{fps} 11 \mathrm{Mpps}$ \\
\hline Sandia (Ref. 4) & Full field & $\mathrm{AM} / \mathrm{FM}$ & $0-1500$ & 150 & Dynamic range (8-bit) & $512 \times 480$ & $9.77 \mathrm{~mm}^{\mathrm{a}}$ & $10 \mathrm{fps} 3.8 \mathrm{Mpps}$ \\
\hline Christie et al. (Ref. 5) & Full field & $\mathrm{AM} / \mathrm{FM}$ & $0.8-1.8$ & 20 & Dynamic range (5-6-bit) & $500 \times 500$ & $10 \mathrm{~mm}^{\mathrm{a}}$ & Not specified \\
\hline Gulden et al. (Ref. 6) & Full field & $\mathrm{AM} / \mathrm{FM}$ & $1-2.4$ & 12 & $\begin{array}{l}\text { Dynamic range or } \\
\text { acquisition time }\end{array}$ & $12 \times 8$ & $416 \mathrm{~mm}^{\mathrm{a}}$ & Not specified \\
\hline
\end{tabular}




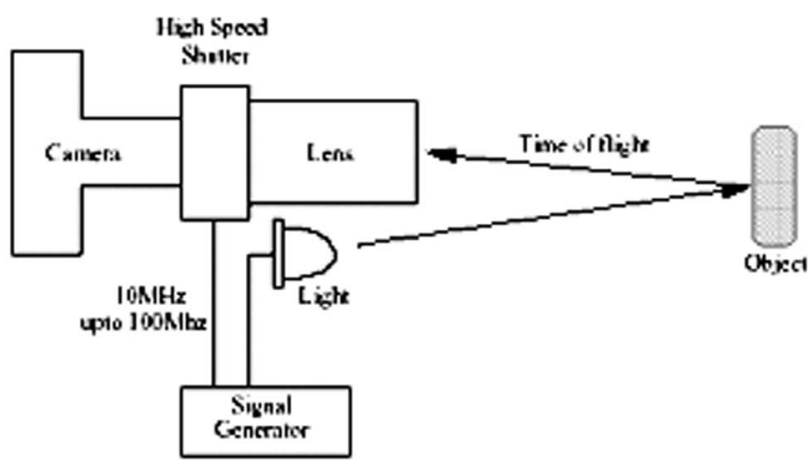

FIG. 2. Diagrammatic illustration of time-of-flight process.

ing from the high speed shutter is of sufficiently low frequency that it can be sampled by standard camera technology without aliasing.

Most described systems (for example, Refs. 5 and 11) use a homodyne configuration, in which the shutter is modulated with the same signal as, and in phase with, the light source. This causes the phase shift due to distance to be encoded into both a very high-frequency component and into the dc component. In these implementations, the highfrequency component is filtered out by the low-frequency response time of the image intensifier used as the high speed shutter, and only the dc component is received at the camera. However, this dc component is contaminated by the reflectance properties of the object, the intensity of the transmitted light as received at the particular point of the scene, and by any ambient background light. Calibration images with the modulation switched off are needed to cancel out the extraneous intensity changes in the dc component that are not due to the phase shift of the time of flight. One could imagine that three or more bits of information could easily be lost in dynamic range due to the varying natural intensities of the scene leaving only four or five bits of information for the encoding of depth due to time of flight if a standard 8-bit camera is used. Consequently, the range resolution of these systems is severely limited by the dynamic range of the camera, and indeed, Christie et al. ${ }^{5}$ report $2 \mathrm{~cm}$ measurement uncertainty when measuring over a range of $0.8-1.8 \mathrm{~m}$.

One can employ conventional radar type techniques for ranging. Gulden et al. ${ }^{5}$ describe using phase-shift-keying (PSK) and frequency-stepped continuous wave (FSCW) with a CMOS camera that has the high speed shutter integrated into the IC. Their system achieved similar results for both methods with a range resolution of approximately 1 to 1.2 $\mathrm{cm}$ over a range of 1 to $2.4 \mathrm{~m}$, but failed to distinguish two objects closer than $0.5 \mathrm{~m}$ in range. Their system is severely limited by the extreme low resolution of the CMOS sensor, and furthermore, to achieve accurate phase-shift-keying requires a shutter with a (difficult to obtain) almost perfect linear response. In FSCW the signal is encoded into a lowfrequency beat signal where the frequency of the beat is related to the phase angle due to time of flight. Accurate measurement of the frequency of such signals is limited by the frequency-time uncertainty principle, which overly limits the resolution of ranging.

We propose a method of ranging with similar hardware as the systems described above, but with the signals to the light source and the high-speed shutter in a heterodyne configuration. Heterodyning is a familiar technique in laser optics and fluorescence lifetime imaging, ${ }^{12-14}$ but, to the best of our knowledge, has never been considered before for fullfield ranging.

In a heterodyne configuration two signals, one a small but constant frequency higher than the other, are used. Let $f_{1}$ be the frequency of the modulated transmitted light and $f_{2}$ $=f_{1}+\delta$, for some small value of $\delta$, be the frequency of the high-speed shutter. The received light after mixing at the shutter is given by (ignoring reflectance and intensity multipliers)

$$
\begin{aligned}
\cos \left(2 \pi f_{1} t+\phi\right) \cos \left(2 \pi f_{2}\right)= & \cos \left(2 \pi\left|f_{1}-f_{2}\right|+\phi\right) \\
& + \text { high frequency terms, }
\end{aligned}
$$

where only the low frequency term on the right-hand side (RHS) is received at the camera. It consists of a beat frequency $\delta=\left|f_{1}-f_{2}\right|$ with a phase shift, $\phi$ namely the phase shift due to the time of flight. Thus to measure the range to a point of a scene imaged in any one particular pixel of the camera, one samples the scene for at least a complete cycle of the heterodyne beat and then calculates the phase of the received signal. It is then trivial to obtain the distance to the scene from the phase.

Heterodyning has a number of significant advantages over previously described methods. The range is encoded into a continuous and easily measured parameter that is not directly limited by digital quantization. The presence of harmonics on the light and shutter signal do not adversely affect the phase measurement, thus nonlinear responses, such as that of the image intensifier, are easily tolerated. As both the dc level and the intensity of the beat signal are not critical in determining phase, this method is naturally tolerant of background lighting and surface reflectivity inconsistencies. Finally, the bandwidth of the electronic driving circuitry need not be wide, thus simplifying the design.

\section{PHYSICAL AND ELECTRONIC CONSTRUCTION}

An overview of the hardware system was presented in Fig. 2. The principle components include a camera system with a variable shutter speed capable of opening and closing at rates in excess of $20 \mathrm{MHz}$, a light source that can be modulated at a similar rate, and a mechanism to capture the images created.

Two frequencies must be generated, $f_{1}$, the frequency of the modulated transmitted light and $f_{2}=f_{1}+\delta$ (where $\delta$ is small), the frequency of the high-speed shutter. To generate these frequency locked signals, two AD9852 Analog Devices digital synthesizer chips interfaced to an embedded controller are used. These digital synthesizer chips use DDS (direct digital synthesis) technology and a high-speed, highperformance $\mathrm{D} / \mathrm{A}$ converter to form a digitally programmable agile synthesizer function. When referenced to an accurate clock source, the AD9852 generates a highly stable, frequency-phase-amplitude-programmable cosine output. Up to 48-bit frequency resolution is provided, so (for example) 1 microHertz tuning is possible with a $300 \mathrm{MHz}$ SYSCLK 


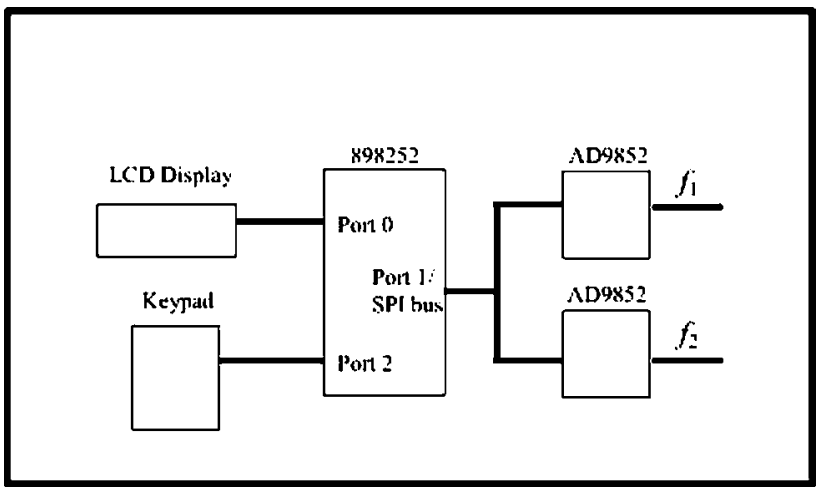

FIG. 3. Creation of two frequency locked ac signals.

(generated by a lower frequency external reference that is multiplied internally by the chip's $4 \times-20 \times$ REFCLK multiplier circuit). Possible output frequencies range from dc to $150 \mathrm{MHz}$ (1/2 SYSCLK).

For this application, the AD9852 is configured to produce a cosine signal of 48-bit frequency accuracy, and 12-bit amplitude accuracy. The phase accumulator, responsible for generating an output frequency, is presented with a 48-bit value from the frequency tuning word (FTW) register. The FTW must be programmed into the AD9852, its value being determined by

\section{FTW $=\left(\right.$ Desired Output Frequency $\left.\times 2^{N}\right) /$ SYSCLK,}

where $N$ is the phase accumulator resolution (48 bits), frequency is expressed in Hertz, and the FTW is a decimal number that is internally rounded to an integer and converted to 48-bit binary format. The output cosine signal is conditioned by a $120 \mathrm{MHz}$ passive low-pass filter before being presented to a header for off-chip application.

The frequency synthesizer chips are interfaced to an Atmel AT89LS8252 ('8252) microcontroller that provides the appropriate FTWs. Although the ' 8252 has a range of features, what is important for this application is that it is an 8-bit microcontroller containing 32 programmable I/O lines, a programmable UART serial channel, and a SPI (serial peripheral interface) interface. The block diagram of this circuit is presented in Fig. 3. To the left of the '8252 microcontroller are the keypad and LCD display, which form the user interface for the AD9852 programming.

On power-up, the user is prompted to enter the base frequency the system is to operate at, and the required frequency difference between the two signals. This information is entered via the keypad, and then converted into two sixbyte values (i.e., 48 bits) that numerically represent each desired frequency. The SPI protocol is initialized, and these 48-bit FTWs are sent to each of the AD9852 chips.

Frequency $f_{1}$ from the top ' 9852 is conditioned by a pair of cascaded National LM7171 operational amplifiers to drive a bank of Agilent HLMP EL series monochromatic (red) LEDs. These LEDs have a $40 \mathrm{pF}$ capacitance and a $20 \mathrm{~ns}$ response time. Our application requires that these LEDs be pulsed to either a clean gating signal or sinusoidal signal. Tests so far have successfully driven these LEDs to $20 \mathrm{MHz}$ sinusoidally.
For our implementation, the camera system comprises a Photek MCP125 Image Intensifier attached to a standard 80$200 \mathrm{~mm}$ focal length Nikon F-mount zoom lens. We employ a three terminal device comprising a low voltage photocathode, a single microchannel plate (MCP) and phosphor screen. Higher speed, and higher gain multiple MCP devices are available that would improve the imaging quality; however the MCP125 suffices for prototyping purposes.

To use the Image Intensifier as a fast electronic shutter, a voltage pulse is applied to the photocathode. The on/off gain/ attenuation ratio is typically greater than $10^{10}$, ensuring that an efficient optical block is created. Specifications for the device indicate that a modulation frequency of $100 \mathrm{MHz}$ could be attained, though to date, we have only tested our system up to $30 \mathrm{MHz}$. A better image resolution with less noise and higher contrast can be obtained by gating the microchannel plate $(\mathrm{MCP})$ rather than the photocathode; however this is problematic at our frequencies of interest as this typically requires a voltage swing of several hundred volts.

Frequency $f_{2}$ from the bottom ' 9852 is amplified and dc shifted to apply a $-3 \mathrm{~V}$ to $1 \mathrm{~V}$ signal to the photocathode of the Image Intensifier. The Image Intensifier output is coupled to a Pulnix TM9701 8-bit digital camera, operating at 29.97 fps, via a $25 \mathrm{~mm}$ fixed focal length lens. The frames sampled by this camera are fed to a Matrox image capture card where they are uploaded to an analysis program coded in MATLAB.

\section{METHODOLOGY}

The linearity and range resolution of the system was investigated over a range of $5 \mathrm{~m}$ in a darkened laboratory environment. The modulation frequency was set at $10 \mathrm{MHz}$, and experiments were conducted with $\delta$ values of 1,2 , and 5 $\mathrm{Hz}$. As the camera is not synchronized to the restart of the heterodyne beat, an arbitrary offset result exists in the obtained ranges. For these trials, this offset problem is resolved by the introduction of a reference object at a known distance. With the limitation of the lens' depth of field, this reference object was moved to three locations $(0.800 \mathrm{~m}, 1.500 \mathrm{~m}$, and $2.500 \mathrm{~m}$ ) adjacent to the optic axis of the camera's view. The ranged object (positioned as close as possible to the camera's optical axis) was then moved from a distance of $5 \mathrm{~m}$ to $1 \mathrm{~m}$ away from the imaging system in $0.250 \mathrm{~m}$ increments. Each range involved a $10 \mathrm{~s}$ acquisition at $29.97 \mathrm{fps}$, and was repeated three times.

We apply a discrete Fourier transform in time separately to each pixel and calculate the angle of the complex quantity in the bin of the Fourier spectrum corresponding to the beat signal. This gives the phase of the beat signal that represents time of flight, from which the range is determined.

The Fourier method is particularly good at detecting the phase of a signal when significant noise is present. ${ }^{15}$ It provides an optimal estimate of phase provided the noise is white and Gaussian and no harmonics are present. ${ }^{16}$ If harmonics are present, as with the hardware described here, the Fourier method does not provide an optimal estimate, however it still provides a reasonably reliable result. ${ }^{15}$

As the camera's frame rate is not an integer multiple of 


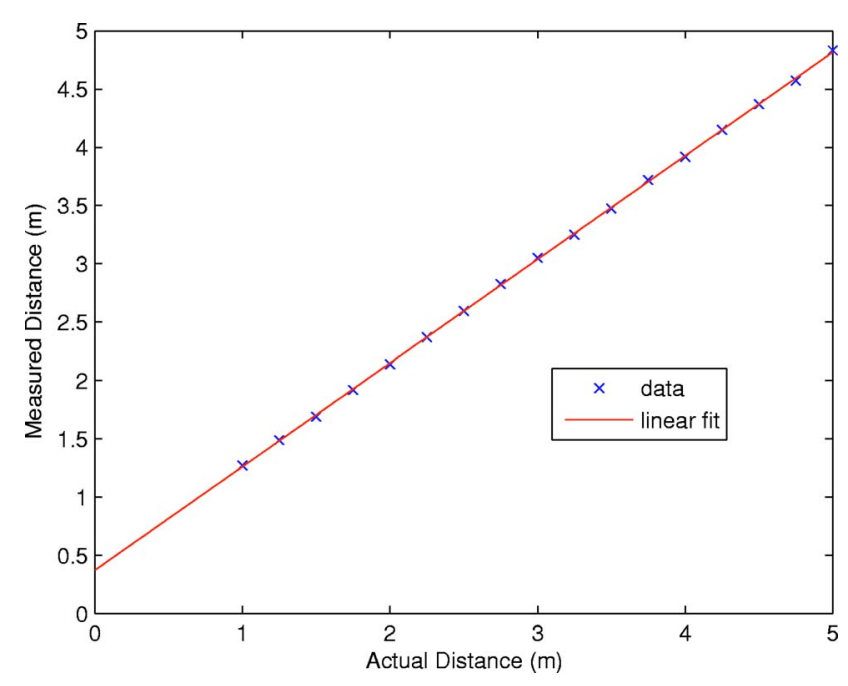

FIG. 4. (Color online) Plot of ranged distance versus actual distance for $\delta=1 \mathrm{~Hz}$.

the modulation signal frequency difference, it is likely that the heterodyne beat frequency will not precisely land on a single bin in the Fourier domain. We therefore have also analyzed the data by zero padding the sequences to eight times their original length before Fourier transforming and then locating the peak in the magnitude of the Fourier spectrum. Quadratic interpolation was used on the bin corresponding to the peak and the two neighboring bins to better locate the peak to subbin accuracy. This frequency estimate was repeated over the 4000 or so pixels with the strongest signal in the sequence and an average taken to give a precise estimate of beat frequency.

The phase was calculated for each pixel by linear interpolation of the phase between the two neighboring bins to the frequency estimate. This provides a range image comprising $768 \times 484$ pixels; however only a round region of about 500 pixel diameter corresponds to the view of the image intensifier.

Each captured data set is processed with the Fourier analysis approach described above. For each of these experiments the camera focus was set to the middle of the range under study. A reference block region of interest (ROI) of area between 1600 and 2500 pixels was used for the reference block for each of the three studies. This ROI was kept the same within a study as the reference block always appears in the same place in each image. The mean and standard deviation of range was calculated over this ROI, and a constant range offset was subtracted from the range image to shift the mean range of the ROI of the reference block to the correct range $(0.800 \mathrm{~m}, 1.500 \mathrm{~m}$ or $2.500 \mathrm{~m})$. Another ROI of 100 pixels was assigned to the moveable block. This ROI was kept the same for each data set of the moveable block over a study. The mean and standard deviation of measured range over the ROI was calculated for the moveable block for each range reconstruction.

\section{RESULTS AND DISCUSSION}

The results are illustrated in Fig. 4. The linearity and repeatability are excellent; however, the gradient is 0.89
TABLE III. Standard deviation of difference between measured and actual range.

\begin{tabular}{cc}
\hline \hline$\delta(\mathrm{Hz})$ & Standard deviation $(\mathrm{cm})$ \\
\hline 1 & 1.2 \\
2 & 1.3 \\
5 & 2.6 \\
\hline \hline
\end{tabular}

rather than the expected 1.00 for all three studies. The data points for each study are made to be accurate for one measured distance. The others will be inaccurate due to the nonunity slope. In order to obtain the continuous slope of Fig. 4, an offset is added to each study.

In principle this anomaly could be calibrated for and removed from the results since the results are consistent and repeatable; however it is of course preferable to identify and remove the source of this error. Thorough checking has confirmed that the measurement and signal generation equipment is well calibrated, that errors due to changing path length of the light signal as one moves off the optical axis of the ranger are far too small to be the explanation, and that the reconstruction algorithm gives a correct slope of 1.0 for simulated data. A theoretical analysis of heterodyning shows that the delayed phase of the signal is dependent on only two parameters, the range to the object and the modulation frequency. We conclude that contamination of the range signal being received at the image intensifier is occurring. Multipath effects can be excluded (light being reflected off some other object, such as a wall, onto the scene) as these increase the measured range. Experiments conducted with extra light blocking material over nearby reflecting objects and inbetween the ranger and the LED light source yield the same resultant slope. It is now believed that the problem is direct electromagnetic inductive coupling between the LED light source and the image intensifier. Theoretical estimations of how measured range is affected by a partial direct coupling of the light source signal to the image intensifier confirms the validity of this hypothesis. The new system (Sec. VI) is being designed with specific shielding in place to eliminate this.

Allowing for the nonunity gradient, it is noted that the error increases with an increase in $\delta$, as illustrated in Table III. All measurements were performed over a 10 second time frame. The larger error in the $5 \mathrm{~Hz}$ data can be explained by the fact that a beat cycle occurs every six frames, compared to every 30 frames for the $1 \mathrm{~Hz}$ case. This reduction in the number of frames results in a corresponding increase in the error of phase resolution and therefore range determination. Padding the data by a factor of 8 prior to Fourier analysis did not significantly alter the determined range.

It should be noted, that although an 8-bit digital camera is employed, the maximum intensity over the region of interest is generally 5 bits or less, depending upon the color of the imaged object. This loss of bit resolution has a dramatic effect on the range resolution. An analysis by Zhao and Surrel $^{17}$ demonstrates that quantizing the intensity data to 6 bits will result in an approximately five time increase in the standard deviation of the phase error compared to an 8-bit 
quantization. This is comparable to the error margins obtained in our results. An increase in the number of bits to 10 reduces this error by more than an order of magnitude (compared to a 6-bit system), and our calculations indicate that at 12-bit quantization our system should have a precision of approximately $1 \mathrm{~mm}$ (for a 100 pixel averaged region).

To conclude the error analysis, consideration should be made to the fact that the error will reduce as the modulation frequency increases. This is explained more fully in Sec. VI, but can clearly be determined from noting that as the modulation frequency increases, the wavelength reduces while the quantization levels remain unchanged. If the modulation frequency was increased from $10 \mathrm{MHz}$ to $100 \mathrm{MHz}$, there would be a corresponding order of magnitude reduction in range determination error.

In summary, the employment of a 12-bit quantization system, running at $100 \mathrm{MHz}$ could provide range measurements with a precision at the submillimeter level (for a 100 pixel averaged region). However, for such resolution to be useful, the camera and lens system would need to be calibrated to account for radial and decentering distortions, the nonlinearity of the irising effects in the image intensifier would also need to be compensated for. Consideration would also need to be given to the geometrical arrangement of the illumination and camera system to compensate for any variations in the path length of the modulated light.

\section{SYSTEM LIMITATIONS AND FUTURE IMPROVEMENTS}

Although the hardware system described in Sec. IV functions as intended, there are two limitations. The first is that the distance determined by this system suffers from an arbitrary offset error due to the $2 \pi$ cycling of the returned signal. For example, using a $10 \mathrm{MHz}$ gating signal, then from

$$
\lambda=\frac{c}{f}
$$

we find a $2 \pi$ cycling of the range measurement over a return path length of $30 \mathrm{~m}$. The consequence of this is that with this single frequency, we would be unable to uniquely determine if an object was 1 meter away, or 16 meters away, there being an unknown distance bias of $0,15,30, \ldots, n$ $\times 15$ meters. Relative distances within this 15 meter window can however, be precisely determined.

To resolve this bias, a sweeping frequency can be applied, and with well-known signal processing techniques, the absolute distance to the object being imaged can be uniquely determined. Alternatively, and preferentially, a dual frequency system could be employed. The low frequency modulation can coarsely determine the range and remove the ambiguity discussed above and the high frequency modulation can provide the fine range determination within this coarse window. An alternative method would be to use a variation in the mean intensity of the reflected light. However, this method relies on the object possessing an invariant color, texture, and orientation as a variation in any of these parameters will result in a variation in the received intensity that is independent of the object's range. We prefer not to restrict the objects we can range to in this fashion, and hence prefer the dual frequency approach.

The second limitation is that with the system designed as described above, there can be a phase difference between $f_{1}$ and $f_{2}$. This phase difference also leads to ambiguity in the absolute resolved difference. To account for this, a second design of the circuit is proposed, using three Analog Devices AD9952 digital frequency synthesizer chips. These ICs offer increased bandwidth over the AD'9852, operate at a lower voltage, and importantly, have only $10 \%$ of the power dissipation. This is achieved at the expense of functionality, many of the '9852's output options are not provided. However, the AD9952 includes synchronization inputs and outputs, allowing three of these devices to be synchronized together.

Two of these devices output $f_{1}$ and $f_{2}$ as before, the third device outputs a synchronized pulse at an integer multiple of the beat frequency $\delta$ that initiates the frame grabbing. This will ensure that the beat frequency is a known bin in the FFT. This modified circuit is currently under construction, and as mentioned, will not affect the relative distances between objects in the 15 meter window, only the absolute resolved distance. There is also the problem that the acquisition cycle is not synchronized to the heterodyne beat, thus, the camera starts its video sequence at an arbitrary point in the heterodyne beat cycle. The new 12-bit camera system being purchased has the capability to be externally synchronized, removing both of these problems.

The Agilent LEDs are not able to be cleanly pulsed at the higher frequency ranges that we expect our system to perform at (eventually between $50 \mathrm{MHz}$ and $100 \mathrm{MHz}$ ). The frequency synthesizer chips are capable of providing the signals in this frequency range, but the light source will have to be changed. Additionally, the total range of our system is limited by the range of the illuminance source and sensitivity of the intensifier/camera. To increase both the modulation speed and the range, it is proposed to investigate laser diodes as a light source (in combination with the more sensitive 12-bit camera), and it is hoped to eventually extend the system's operating range to $100 \mathrm{~m}$.

To operate in outside environments, interference filters are proposed to block the ambient illumination, and receive primarily the modulated light signal. It is expected that strong ambient sunlight might limit the range of the system and it will also affect the lower significant digits of the quantized range result (depending upon the intensity of the light that reaches the $\mathrm{CCD}$ ). Finally the limitations discussed at the conclusion of Sec. VI must be accounted for.

\footnotetext{
${ }^{1}$ J. Busck and H. Heiselberg, Appl. Opt. 43, 4705 (2004).

${ }^{2}$ Z-Cam http://www.3dvsystems.com/products/zcam.html

${ }^{3}$ G. Yahav and G. J. Iddan, USA Patent No. 6,100,517 (2000).

${ }^{4}$ T. C. Monson et al., Proc. SPIE 3707, 409 (1999).

${ }^{5}$ S. Christie et al., Meas. Sci. Technol. 6, 1301 (1995).

${ }^{6}$ P. Gulden et al., IEEE Trans. Instrum. Meas. 51, 679 (2002).

${ }^{7}$ L. R. Gabello et al., USA Patent No. 6,584,283 (2003).

${ }^{8}$ G. J. Iddan and G. Yahav, Proc. SPIE 4298, 48 (2001).

${ }^{9} \mathrm{~T}$. Oggier et al., presented at the OPTO-Conference, Nürnberg, Germany, 2004.

${ }^{10}$ B. Stann, W. C. Ruff, and Z. G. Sztankay, USA Patent No. 5,877,851 (1999).

${ }^{11}$ M. W. Scott, USA Patent No. 4,935,616 (1990).
} 
${ }^{12}$ R. Cubeddu et al., J. Phys. D 35, R61 (2002).

${ }^{13}$ M. Christenson and S. Sternberg, in SPIE's oemagazine, 2004, p. 28.

${ }^{14}$ E. M. Sevick-Muraca and D. Y. Paithankar, USA Patent No. 5,865,754 (1999).
${ }^{15}$ P. O'Shea, in Electrical Measurement, Signal Processing and Displays, edited by J. G. Webster (CRC Press, Boca Raton, FL, 2004), p. 5.

${ }^{16}$ D. Rife and R. Boorstyn, IEEE Trans. Inf. Theory 20, 591 (1974).

${ }^{17}$ B. Zhao and Y. Surrel, Appl. Opt. 36, 2070 (1997). 